\title{
Research on positioning method of industrial wireless sensor networks
}

\author{
Qingjun Yang ${ }^{1,3}$, Dongsheng $\mathrm{Ji}^{2^{*}}$, Yukai Yao ${ }^{2}$, Enzhan Zhang ${ }^{2}$ and Xiaoyun Chen ${ }^{*^{*}}$
}

\begin{abstract}
Location data associated with wireless sensor networks represents critical information. Current developments in localization algorithms employed in industrial wireless sensor networks seek to adopt positioning methods that are more robust and stable, and more accurate and efficient while requiring a minimum of resources. Therefore, the present study capitalizes on the industrial wireless sensor network that has a strong correlation between applications to propose a positioning method design based on a Monte Carlo localization algorithm that is ideally suited to the narrow channel environment encountered in underground mining activities. The proposed positioning method offers very low computational complexity, which greatly reduces the use of network resources. Simulation experiments demonstrate that the proposed Monte Carlo localization algorithm provides strong stability and relatively high positioning accuracy.
\end{abstract}

Keywords: Localization method, Industrial wireless sensor networks, Narrow channel

\section{Introduction}

Wireless sensor networks (WSNs) have a number of advantages, such as low cost, simple deployment, and network structures featuring self-organization properties and dynamic characteristics. As a result, WSNs are widely adopted in numerous applications, such as military, medical, environmental monitoring, logistics and transportation, and space exploration. Positioning technology represents a critical application of WSNs, where objects within the sensor field are located according to network connectivity, rather than by physically measuring distances and angles. However, sensor network node location information is essential in most positioning applications because coherent positioning within the sensor field cannot be obtained under a lack of node location data. While a number of advanced node localization algorithms have been developed, such as the distance vector hop (DV-Hop) algorithm [1, 2], convex programming $[3,4]$, and multi-dimensional scaling mapping (MDS-MAP) [5], these algorithms require a centralized location. As such, positioning

\footnotetext{
* Correspondence: jids8012@163.com; chenxy@|zu.edu.cn

${ }^{2} \mathrm{~S} c h o o l$ of Computer and communication, Lanzhou University of

Technology, Lanzhou 730000, China

'School of Information Science\& Engineering, Lanzhou University, Lanzhou

730000, China

Full list of author information is available at the end of the article
}

technology employing WSNs still requires further development. Positioning applications of WSNs are particularly critical in mining environments. Here, the detailed real-time location of personnel and equipment is essential to strengthen management operations and reduce accidents. However, GPS positioning devices are prohibitively expensive and cannot be used underground. Underground positioning applications must employ node localization algorithms that are relatively simple to implement, are suitable for long-term use, and provide stable positioning results with high precision. In response to the requirements of this challenging environment, the present work proposes a narrow channel Monte Carlo localization (NCMCL) algorithm.

\section{Problem description}

Coal mines employ narrow roadways, where the roadway width (i.e., $2-10 \mathrm{~m}$ ) is generally much less than the length of the roadway. For example, the Shitanjing mining face of the Shenhua Ningxia Coal Industry Group Co. employs two full-length roadways back to the wind tunnels that are $692 \mathrm{~m}$ in length and $3 \mathrm{~m}$ in width [6]. For WSN positioning, anchor nodes of known position are uniformly deployed in the roadway and are fixed at one side of the mine tunnel. By equipping personnel and equipment with network 
nodes, the positions of personnel and equipment can be tracked in real time relative to the positions of anchor nodes based on the location data transmitted to the management center.

\section{Existing research}

The Monte Carlo localization (MCL) method for WSNs is a posterior probability model with very good positioning accuracy and efficiency. Monte Carlo localization prediction method is conducted by repeating two steps to continuously update the position of mobile nodes. These include a prediction step, where the sampling point location of a mobile node updates the sample points and calculates the position of the filter updates. $\mathrm{Hu}$ and Evans proposed an MCL algorithm [7] that provided a stable orientation, but its sampling and downhole environment misplaced process necessitated a high degree of computational complexity. Subsequently, Baggio and Langendoen proposed a Monte Carlo localization boxed (MCB) positioning method [8] that made full use of the two-hop node collar ranks, and provided enhanced positioning accuracy and reduced computational complexity relative to earlier methods. However, their misplaced methods are ineffective in an underground environment, and the computational complexity remains excessive. To accommodate this particular environment, the above two methods were combined to propose the center MCL algorithm, which takes full advantage of high-precision Monte Carlo methods in conjunction with a simple positioning method, in addition to the underground location when the effect is not obvious and there is no part of the effect.

The application environment is extremely relevant to the performance of a WSN localization algorithm, where each localization algorithm has its own characteristics, and no single algorithm can guarantee optimum performance under all conditions [9]. Moreover, the performance of a given positioning algorithm can vary widely under different application environments. Therefore, the design of WSN localization algorithms must be conducted in conjunction with the actual environment of the application.

Both MCL and MCB positioning methods adopt stochastic models of nodes moving in three dimensions. Another proposed method [10] is based on the motion before the node position prediction node to locate several points, but only roadway in both directions node only one direction to move in two directions. Therefore, positioning than MCL, MCB, and the positioning method in [10] are more simple random in both directions. Each location algorithm-based MCL node location is required to obtain a sample, and then filtered sample coordinates are not anchor node signals overlapping coverage areas point. Algorithms then look at whether there is enough of a sample point. If the sample is not enough, repeat the sampling point and expand the sampling region. Finally, the positions of the nodes are calculated according to the weight of the sample points. These methods specific to a single-node computation mine were a bit big and are therefore not conducive to the long-term use of nodes. The roadway sampling area is itself small, and a paper filter that eliminates the need for the sampling step in the practical application of these algorithms might be more effective.

A WSN localization algorithm requires ranging divided into two categories based on the positioning and orientation ranging without ranging. Location-based distances are determined by measuring the distance between nodes and the angle information between nodes, and then trilateral ranging, triangulation, or maximum likelihood estimation methods are employed to locate the node itself. Typical distance-based localization algorithms include received signal strength indicator (RSSI) [11], time of arrival (TOA) [12], time difference of arrival (TDOA) [13], and angle of arrival (AOA) [14, 15]. Ranging technology based on location positioning has particular advantages, particularly in low-power, lowcost applications.

\section{Proposed algorithm}

WSN localization algorithms are shorter than the $\mathrm{MCB}$ and MCL time higher positioning accuracy. MCB has higher sampling and filtering efficiency than MCL because the sampling region adopted by $\mathrm{MCB}$ has a smaller area than that adopted by MCL. In addition, the reason why MCB filter sampling point is because when there is less anchor node sampling area out of a portion of the anchor node of the signal will overlap the coverage area. In $\mathrm{MCB}$, the mobile node can receive signals from anchor nodes outside the area covered by each to form a rectangle. Then, the area-circumscribed rectangle is covered by a section of all other anchor boxes, usually in addition to the anchor box anchor signal node mutual coverage area outside the region, as well as some regional or no signal coverage area with only a few anchor nodes. Therefore, the MCB position still requires filter methods. This paper considers that targeting efficiency will be greatly improved if the sampling area lies inside the overlapping coverage areas of anchor node signals, such that filtration and repeated sampling can be completely eliminated. The proposed algorithm is presented as follows.

Figure 1 illustrates the anchor node distribution of a narrow mine roadway of width $w$, where $\mathrm{A}$ and $\mathrm{B}$ are two anchor nodes separated by a distance $d$, with an equivalent node communication radius $r$. The anchor nodes are separated by cloth on the side of the roadway, 


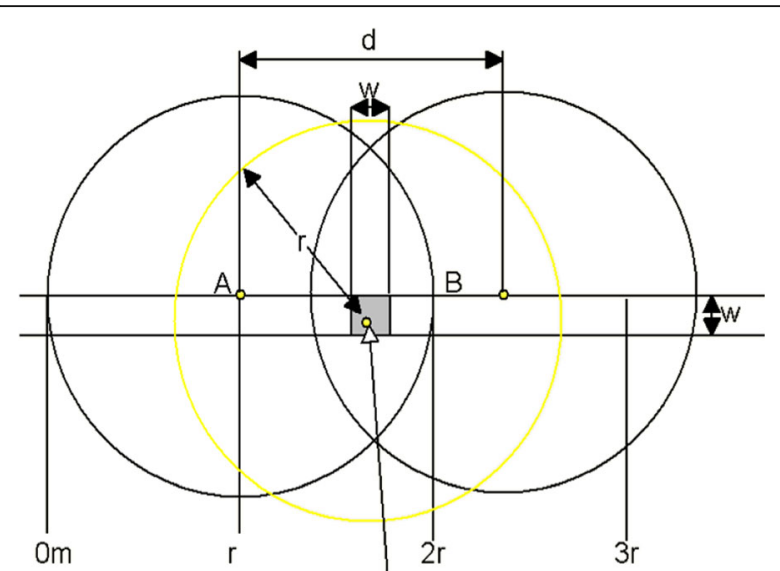

Fig. 1 Anchor box node distribution. The mine has a narrow roadway, roadway width $\mathrm{w}, \mathrm{A}$ and $\mathrm{B}$ two anchor nodes, the node communication radius $r$, anchor nodes separated by cloth on the side of the roadway $d, d$ less than $2 r$ that any two anchors inter-node communication radius overlap; the anchor box width is limited to the roadway width, length is limited to the roadway width

and the inter-node communication radius of the two anchors overlap for $d<2 r$. In addition, the anchor box length and width are limited by the value of $w$. A node between the two anchors festival movement will form the anchor box (with collar ranks hop node of the current node as a reference to calculate the center of these nodes in three positions, depending on the location, the center coordinates origin plus up and down each to half the width of the roadway, forming the anchor box boundary), i.e., in the vicinity of the anchor node to the anchor node as an anchor formed in the center box $\mathrm{C}$, near the center when the two anchor nodes will be the center point coordinates of the center to form an anchor box. If an anchor box region is accordingly defined, the maximum error of locating a mobile node in the middle of the anchor box is $\varepsilon=d / 4+w / 2$.

For example, setting $r=50 \mathrm{~m}, w=5 \mathrm{~m}$, and $d=25 \mathrm{~m}$, resulting in four anchor nodes being laid within $100 \mathrm{~m}$, mobile nodes will form an anchor box at seven locations when moving over this 100-m distance. Therefore, according to the previously stated formula for $\varepsilon$, the theoretical maximum possible location error of mobile nodes is less than $\varepsilon=25 / 4+5 / 2=8.75 \mathrm{~m}$, which will be smaller during actual positioning. The numbers of anchor boxes formed by mobile nodes over a total $100-\mathrm{m}$ distance are listed in Table 1 for a varying number of anchor nodes. Analysis indicates that the number of anchor boxes can be defined as (roadway interval length/anchor nodes +1$)+($ roadway length/anchor node spacing +1$) / 2$. After the formation of an anchor box, the box in which the anchor points were samples. If the current neighbor node list is empty, then
Table 1 Number of anchor boxes formed by mobile nodes over a total 100-m distance

\begin{tabular}{lll}
\hline $\begin{array}{l}\text { Number of anchor } \\
\text { nodes }\end{array}$ & $\begin{array}{l}\text { Anchor node } \\
\text { density }\end{array}$ & $\begin{array}{l}\text { Number of anchor boxes } \\
\text { formed }\end{array}$ \\
\hline 2 & 1 & 3 \\
3 & 2 & 5 \\
4 & 4 & 7 \\
\hline
\end{tabular}

the number of sample points obtained during the last sampling is examined. If the number is not zero, the estimated positions of the last places as mobile nodes position; otherwise, the mobile nodes within communication range last no anchor nodes, and the location of mobile nodes is placed at the geometric center of the anchor box. Here, the neighbor node list is empty only if the anchor nodes separated by $100 \mathrm{~m}$, laying on the side of the roadway will produce, namely, the midpoint between the two mobile nodes and anchor nodes on the other side of the roadway. Then, mobile nodes from two anchors the distance between the nodes will exceed the value of $r$ and receive signals. When the anchor nodes are less than 100-m placement, a mobile node in the neighbor node list will include at least one anchor node. Compared to MCL and $\mathrm{MCB}$, the proposed approach can generate a long anchor box and offers good sample collection without worrying too much. As a result, the approach is able to determine the coordinates of mobile nodes down into the accelerated speed of positioning, thereby reducing the computational complexity.

Because mobile nodes can change positions constantly, this method must be repeatedly run, considering this unknown reference anchor node can receive signals hop anchor nodes. Localization of a mobile node is illustrated in Fig. 2, where anchor nodes are static nodes whose locations are known, and the mobile node is a dynamic node whose location is not known, but which can calculate its own position by receiving coordinate data from the anchor nodes.

The computational procedure employed by the proposed NCMCL algorithm can be defined as follows.

\subsubsection{Prediction stage}

(1) First, update the neighbor node list and calculate the coordinates of the center-hop neighbor list. If there are distance-hop neighbor nodes and mobile nodes that are less than a quarter of the distance between anchor nodes to coordinate the neighbor node as the center coordinates, the center coordinates of the origin of the left and right half of the width of the roadway to the applied to the width of the roadway width for the anchor box, forming the anchor box border. 


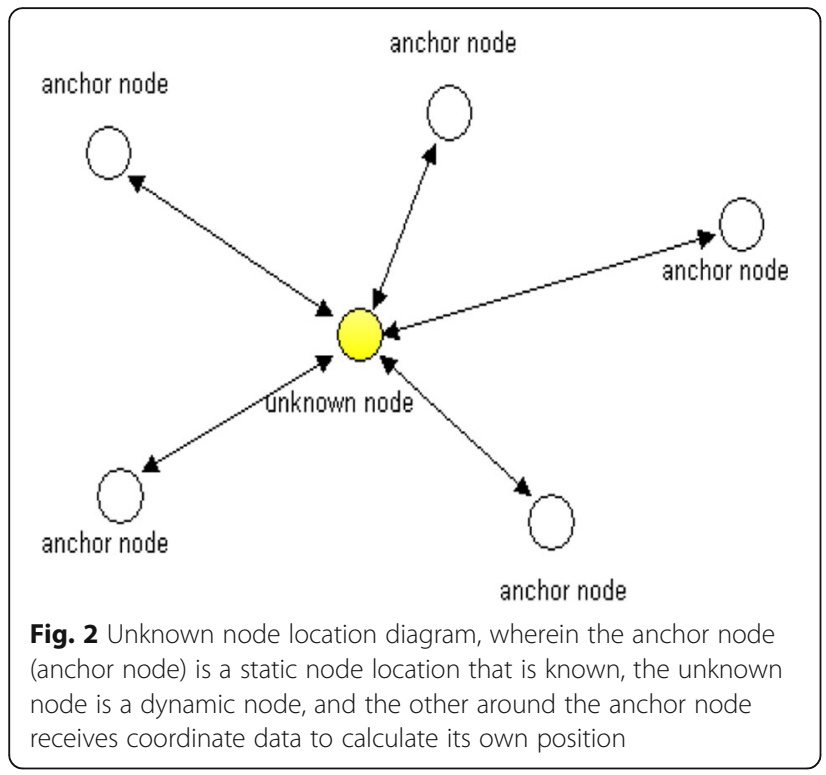

(2) Extract from the anchor box 50 sample points.

\subsubsection{Update stage}

(3) If the current sample number is not zero, execute step 6; if seen that the previous points is 0 , the number of samples is executed by step (4); otherwise, perform step (5).

(4) The geometric center of the area is taken as the current position of the mobile node, as obtained in step (1).
(5) More times the estimated location as the current location of mobile nodes, execute.

(6) Take all the geometric centers of the sample points as the current position of the mobile node, as obtained in step (1).

Communication radii shown in Fig. $1 \mathrm{~A}-\mathrm{C}$ are the anchor nodes separated by a $50-\mathrm{m}$ cloth on one side of the roadway; the node is $50 \mathrm{~m}$.

(1) Using the current mobile node hop collar ranks anchor node (refer anchor nodes within the communication range of the current node, hop collar ranks anchor nodes, and unknown nodes in (Fig. 2) to calculate the geometrical center of these anchor nodes.

(2) To the geometric center of the midpoint of each side plus half the width of the roadway, down to the roadway width of the border, the Cogo center is defined as (centX, centY), which is obtained as follows.

$$
\text { centX }=\frac{1}{n} \sum_{i=1}^{n} A x_{i} \text { centY }=\frac{1}{n} \sum_{i=1}^{n} A y_{i}
$$

Here, $n$ is the current number of living mobile node hop collar anchor node, and $x_{i}$ and $y_{i}$ are the $i$ th $(i=1$,

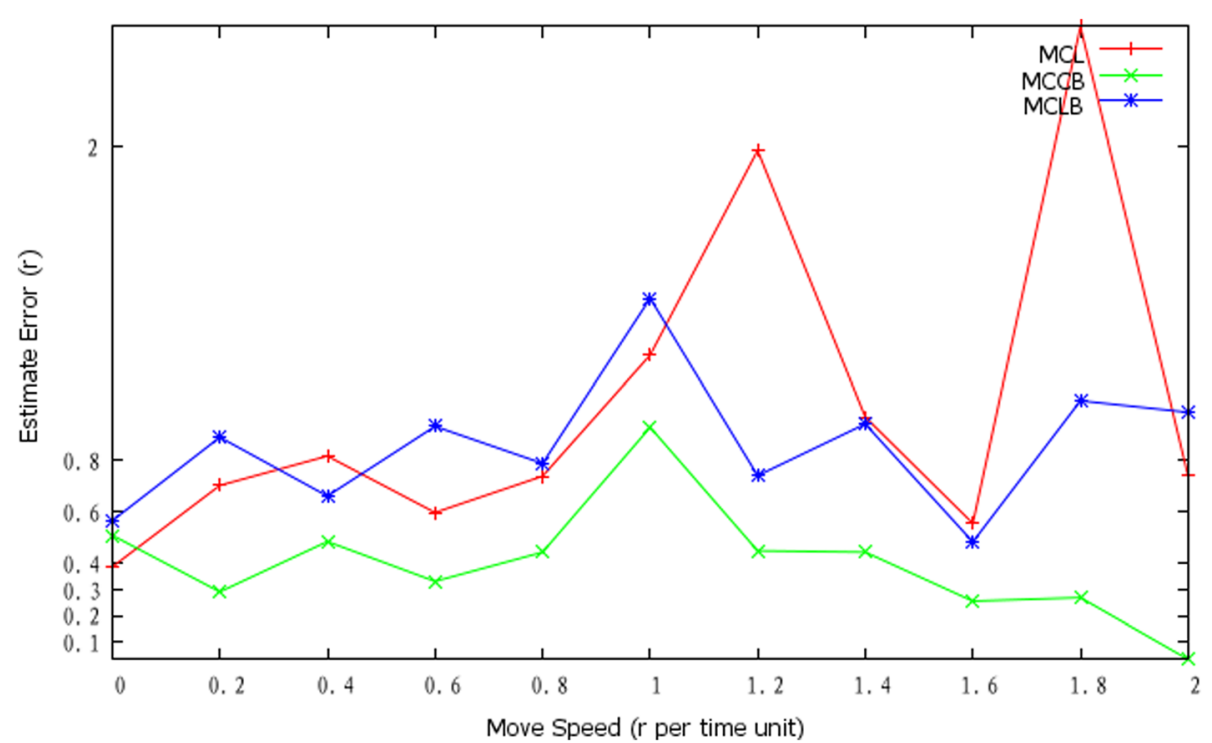

Fig. 3 Anchor nodes by 100-m intervals comparison chart. From the top anchor node, the spacing was $100 \mathrm{~m}$, chart to see when a node moving speed changes from $0 \mathrm{~m}$ to $100 \mathrm{~m}$ accuracy of $\mathrm{CMCL}$ positioning is the best; when you can see the positioning accuracy is approximately $100 \mathrm{~m}$ about $50 \%$; when the accuracy of $30 \%$; and $25 \mathrm{~m}$ accuracy when completely in $17 \%$ or less 


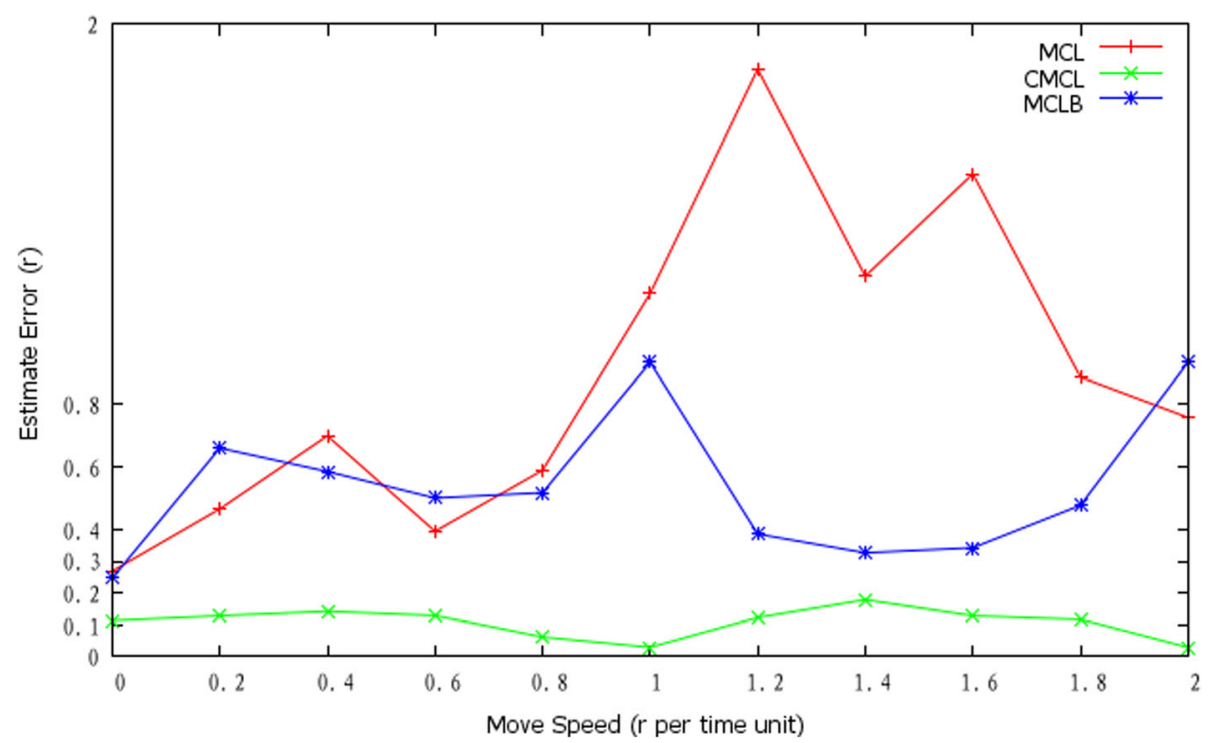

Fig. 4 Anchor node by 50-m interval comparison chart. From the top anchor node, the spacing was $50 \mathrm{~m}$, charting to see when a node's moving speed changes from $0 \mathrm{~m}$ to $100 \mathrm{~m}$ accuracy, in which CMCL positioning accuracy is the best; when the positioning accuracy is approximately about 50\%; when the accuracy is less than $50 \mathrm{~m}$ accuracy; and when completely in $17 \%$ or less

$2, \ldots, n) x$ and $y$ coordinates of the anchor node. Accordingly, cent $X$ and cent $Y$ define the anchor box boundary as follows.

$$
\begin{aligned}
& x_{\min }=\text { centX } \text {-tunnel } \\
& x_{\text {max }}=\text { cent } X+\text { tunnel }_{\text {width }} / 2
\end{aligned}
$$

(3) Here,tunnel width $_{\text {is }}$ the width of the current roadway.

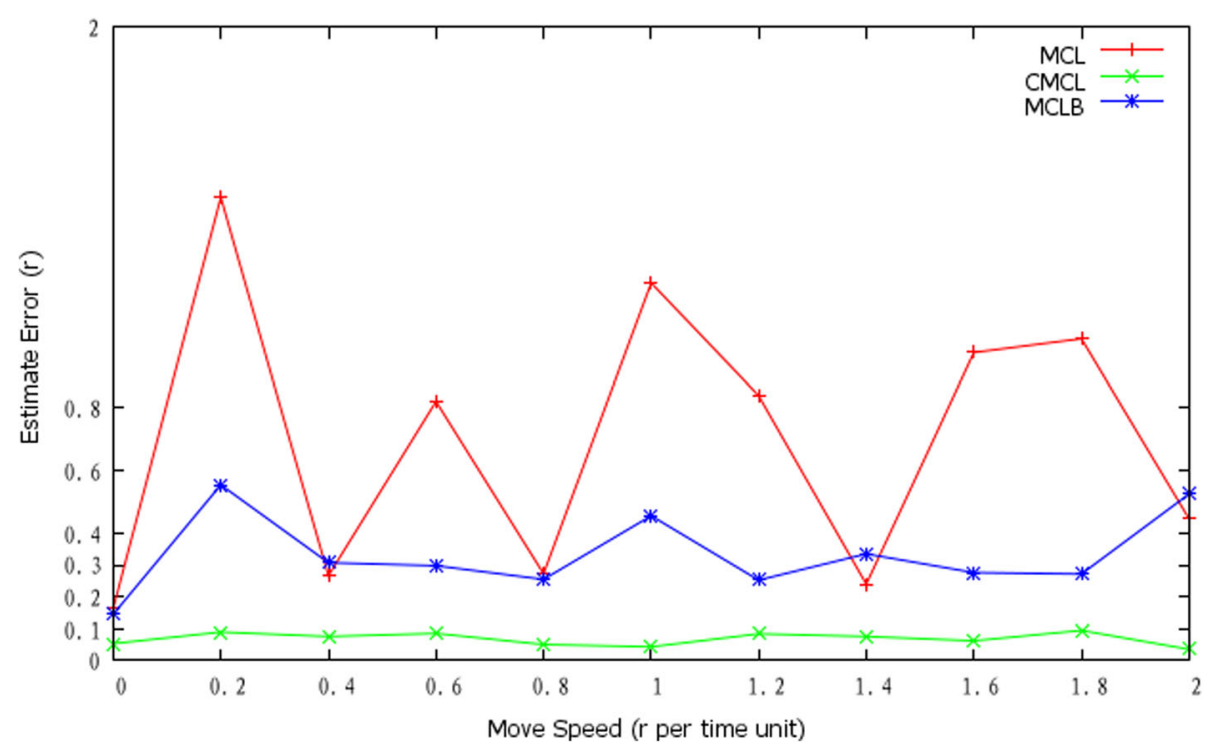

Fig. 5 Anchor node by 25-m interval comparison chart. From the top anchor node, the spacing was $25 \mathrm{~m}$ and $10 \mathrm{~m}$; charting to see when a node moving speed changes from $0 \mathrm{~m}$ to $100 \mathrm{~m}$ accuracy; when you can see the positioning accuracy is approximately $25 \mathrm{~m}$ at about $25 \%$; and when the accuracy is less 


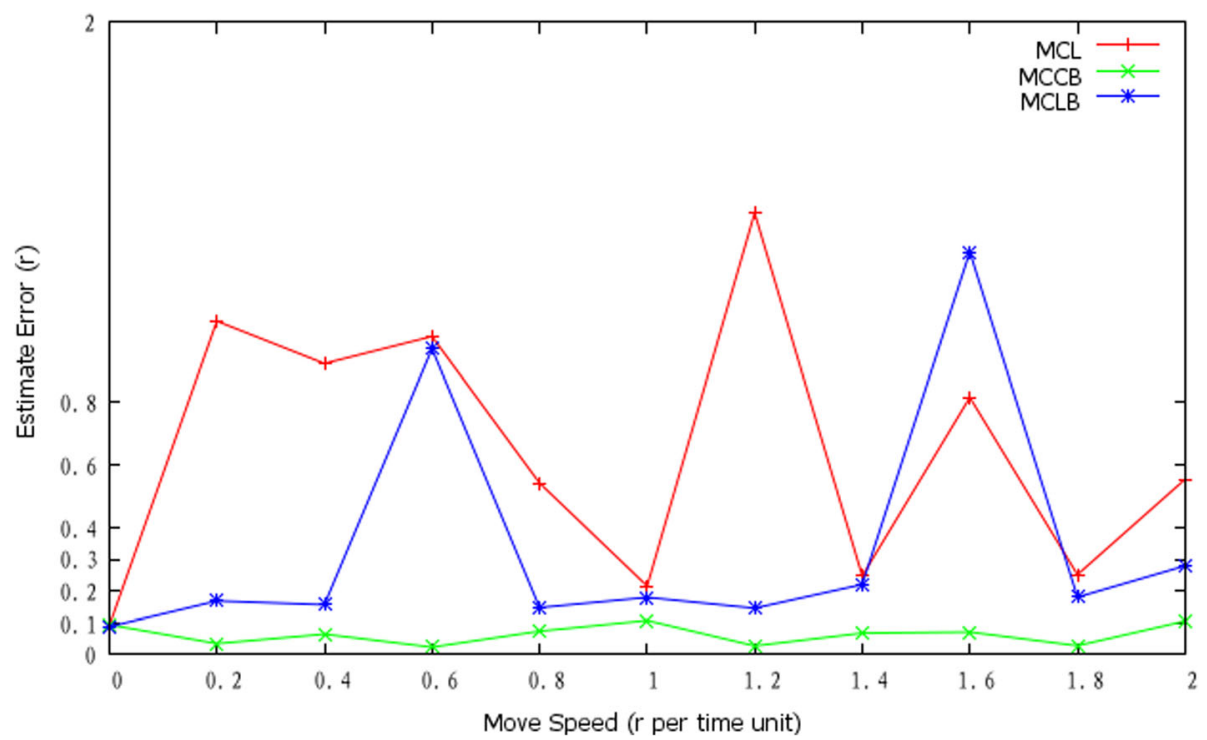

Fig. 6 Anchor node by 10-m interval comparison chart. From the top the anchor node, the spacing was $10 \mathrm{~m}$ in the comparison chart; when the node's moving speed changes from $0 \mathrm{~m}$ to $100 \mathrm{~m}$ accuracy, in which CMCL positioning accuracy is the best; when the positioning accuracy is approximately $100 \mathrm{~m}$ at about $50 \%$; when the accuracy of $50 \mathrm{~m}$ at $30 \%$ or less, $25 \mathrm{~m}$ accuracy when completely in $17 \%$ or less, and $10 \mathrm{~m}$ at $10 \%$ or less

\section{Experiments and discussion}

The localization performance of the NCMCL algorithm was evaluated using the MCL-simulator tools developed by $\mathrm{Hu}$ and Evans for Monte Carlo-based methods [7], and the results obtained were compared with the results obtained using MCL and MCB under equivalent conditions.
In the simulation environment, the length of the roadway was $1000 \mathrm{~m}$, and the value of $w$ was set to various values, including $3 \mathrm{~m}, 5 \mathrm{~m}$, and $10 \mathrm{~m}$, while the value of $d$ was set to various values, including $10 \mathrm{~m}$, $20 \mathrm{~m}, 25 \mathrm{~m}, 40 \mathrm{~m}, 50 \mathrm{~m}$, and $100 \mathrm{~m}$, and $r$ was fixed at $50 \mathrm{~m}$. An unknown number of mobile nodes moved along the roadway randomly in a single direction, and

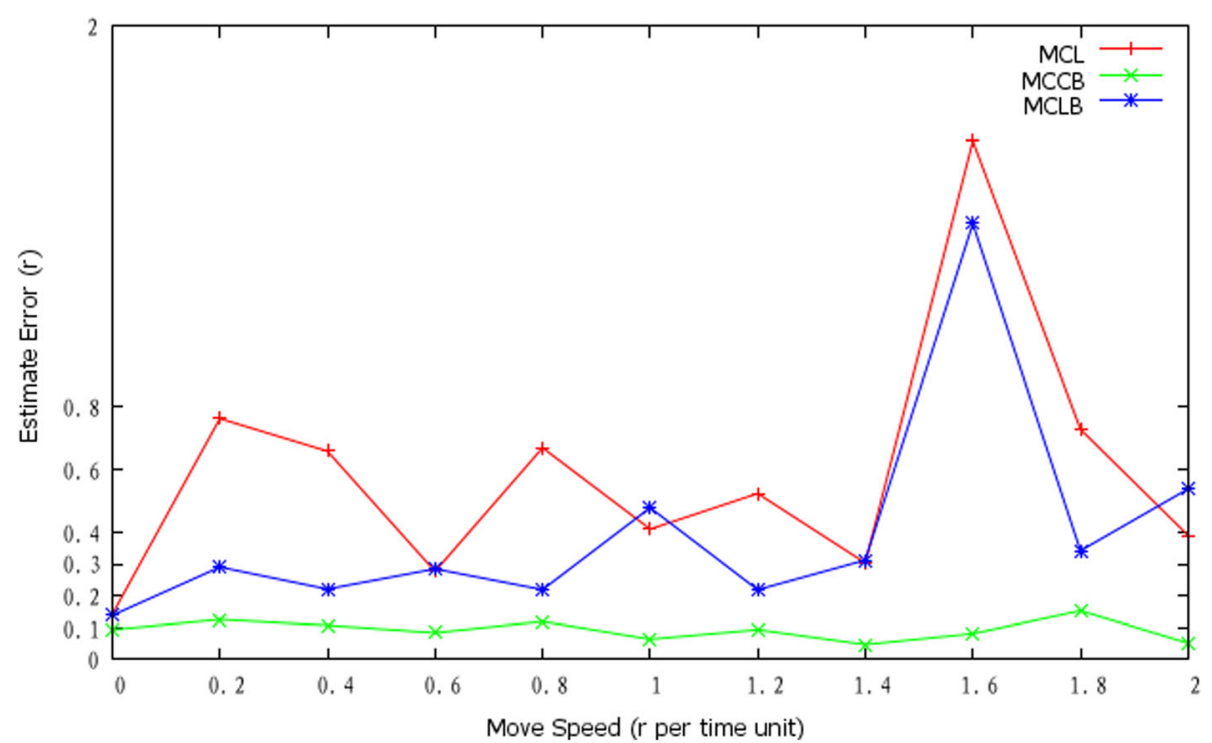

Fig. 7 Roadway width of $3 \mathrm{~m}$ comparison chart. For roadway width at $3 \mathrm{~m}$ under conditions, MCCB precision accuracy is still the best. It can be seen that coverage area is much larger than the width of the roadway, and with the roadway width becoming smaller, the positioning accuracy of the positioning method has all the mentioned precision at about $10 \%$ 


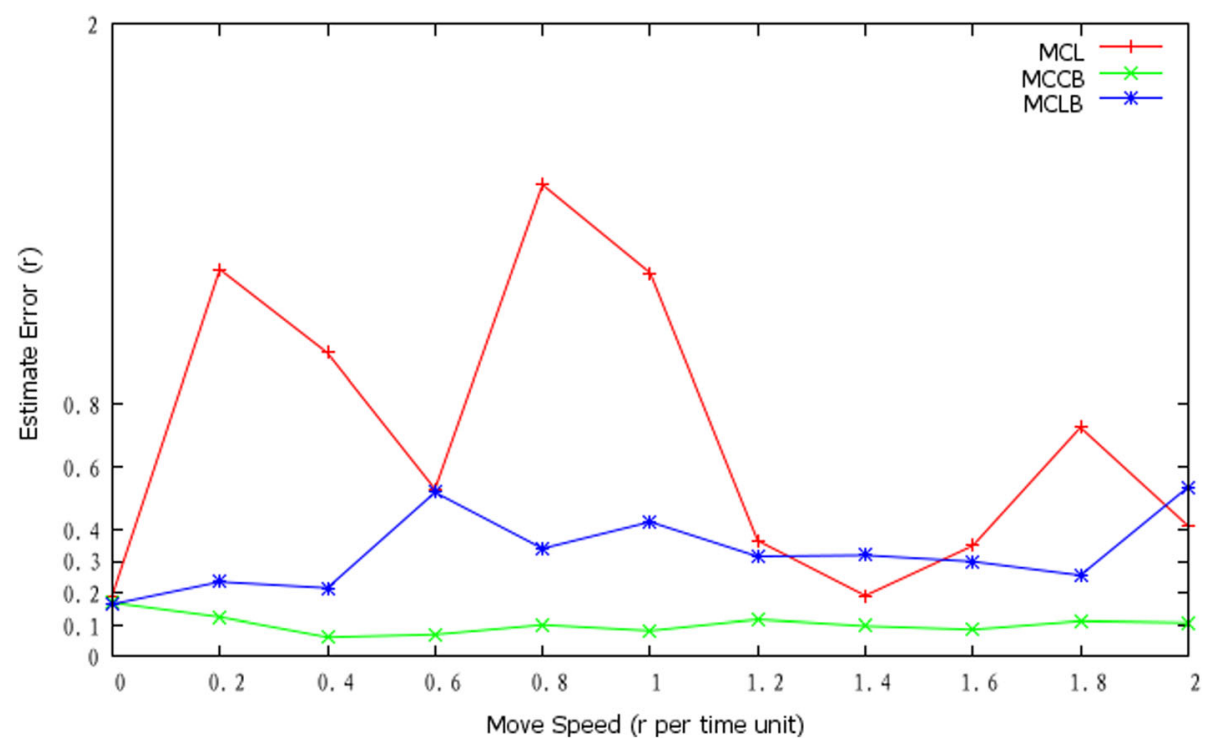

Fig. 8 Roadway width at $5 \mathrm{~m}$ comparison chart. For a roadway width at $5 \mathrm{~m}$ under conditions, MCCB precision accuracy is still the best. As can be seen, the coverage area is much larger than the width of the roadway, and with the roadway width becoming smaller, the positioning accuracy of the positioning method has all the mentioned precision at about $10 \%$

then moved in the opposite direction when arriving at one of the two endpoints of the roadway. The initial speed of motion increased from $0 \mathrm{~m}$ per time interval to a maximum speed of $2 r \mathrm{~m}$ per time interval. The number of samples was uniformly set to 50 sample points.
The localization errors $(\varepsilon / r)$ are obtained for $w=5 \mathrm{~m}$, and $d$ values of $100 \mathrm{~m}, 50 \mathrm{~m}, 25 \mathrm{~m}$, and $10 \mathrm{~m}$ are given in Figs. 3, 4, 5, and 6, respectively. We note that the NCMCL positioning accuracy was substantially better than those of either MCL or MCB, particularly with decreasing $d$. We also compared the positioning accuracy

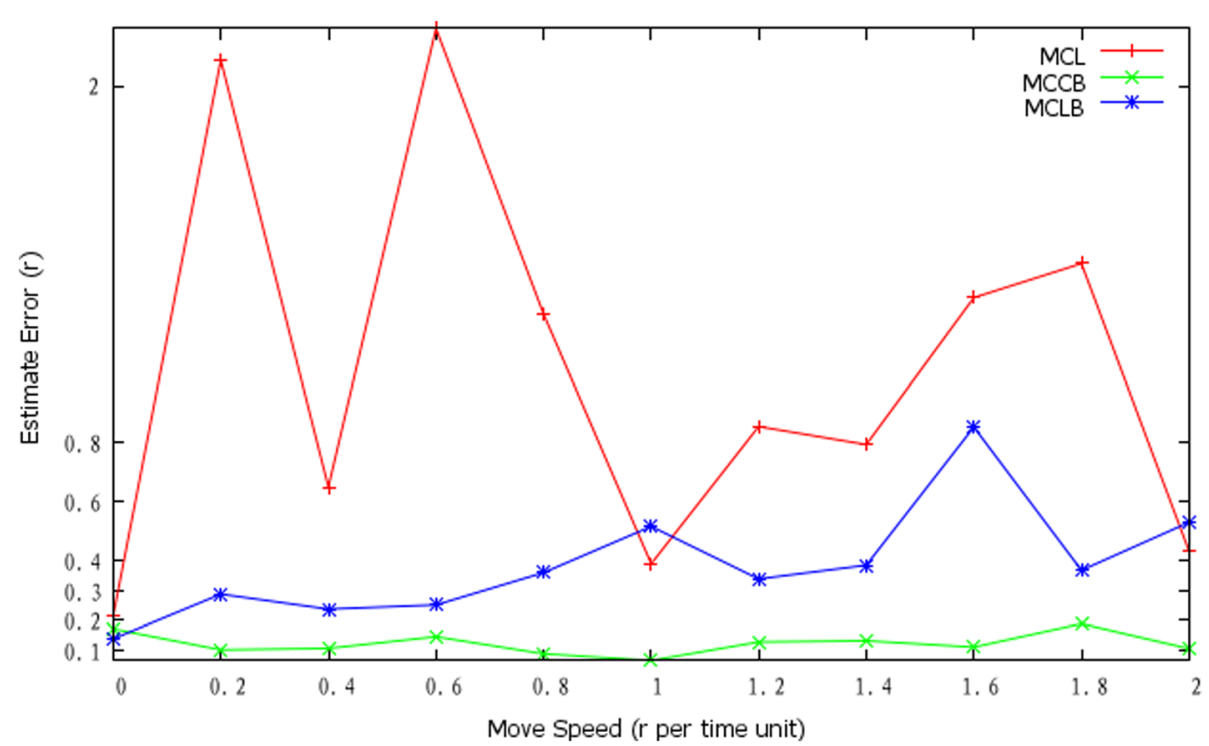

Fig. 9 Roadway width at $10 \mathrm{~m}$ comparison chart. For roadway width at $10 \mathrm{~m}$ under conditions, MCCB precision accuracy is still the best. It can be seen that the coverage area is much larger than the width of the roadway, and with the roadway width becoming smaller, the positioning accuracy of the positioning method has all the mentioned precision at about 10\%. Comparing positioning accuracy under different conditions of the roadway width, the roadway length is fixed at $1000 \mathrm{~m}$; roadway widths are set to $3 \mathrm{~m}, 5 \mathrm{~m}$, and $10 \mathrm{~m}$; and the anchor node spacing is fixed at $25 \mathrm{~m}$ 


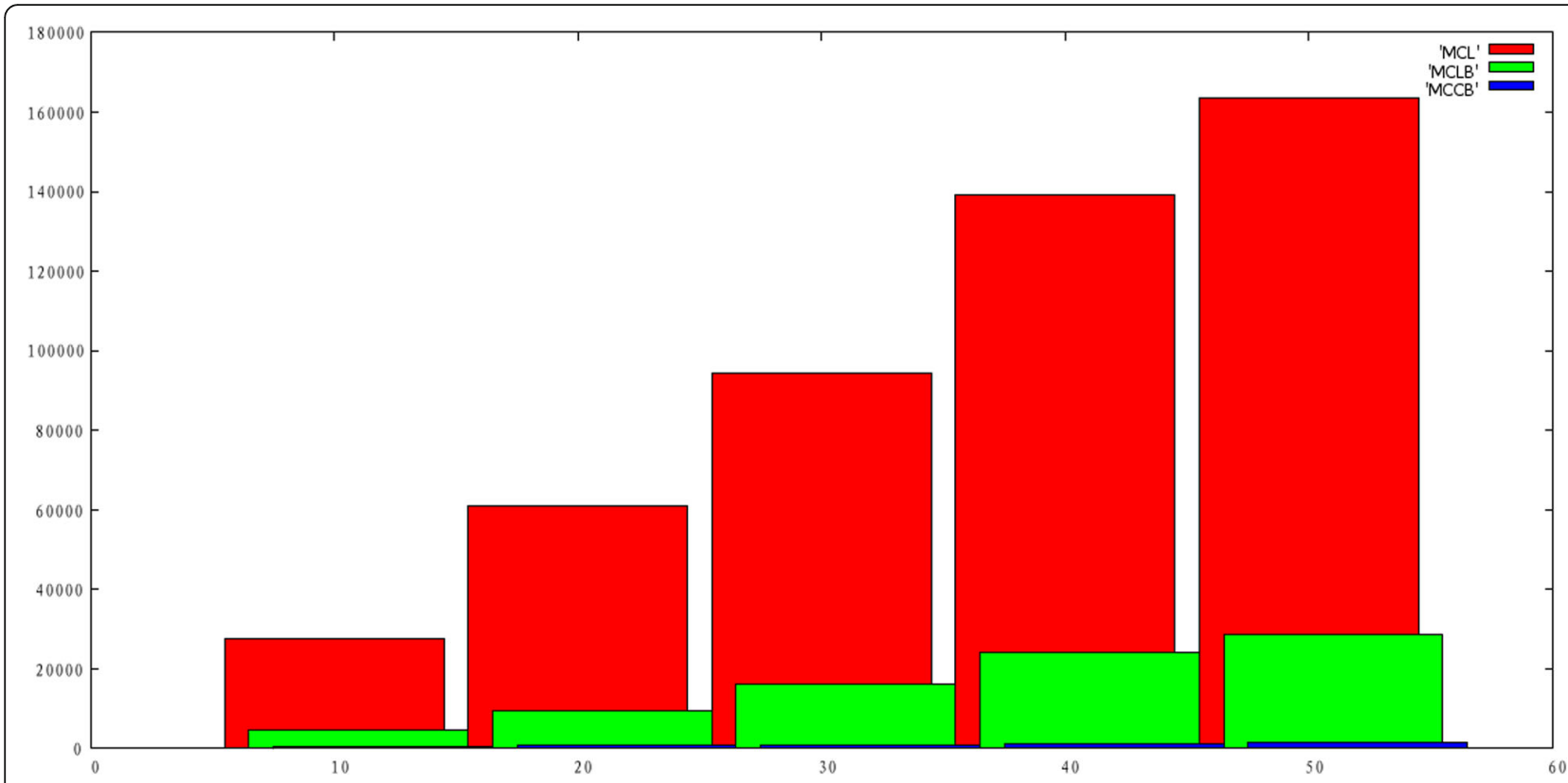

Fig. 10 Algorithm running time comparison chart. The red is for $M C L$ positioning uptime, green for MCB positioning uptime, and blue $\mathrm{CMCL}$ positioning uptime. As can be seen, $\mathrm{CMCL}$ is much smaller than the other two positioning methods

obtained for $d=25 \mathrm{~m}$ under different $w$ values of $3 \mathrm{~m}$, $5 \mathrm{~m}$, and $10 \mathrm{~m}$ in Figs. 7, 8, and 9, respectively. Again, we note that the positioning accuracy of the proposed NCMCL method is considerably better than that of MCL and MCB at all values of $w$ considered. It can be seen that the coverage area is much greater than the value of $w$ as $w$ decreases.

The positioning times required for the NCMCL, MCL, and MCB localization methods are compared in Fig. 10 for $w=5 \mathrm{~m}, d=25 \mathrm{~m}$, and with an unknown number of mobile nodes set as $10,20,30,40$, and 50 . As can be seen from the figure, NCMCL requires a small fraction of the processing time required by the other two positioning methods. We note that MCL was particularly slow. Here, MCL extracted from a hop collar communication area ranks among the anchor node samples, and each sample must be drawn with a hop and two-hop anchor nodes to filter the sample extracts 20,000 times in the worst case. Moreover, obtaining a sufficient number of samples is not guaranteed.

\section{Conclusions}

\subsection{Algorithm comparison}

In addition, while MCB has reduced the sampling area requirements, which facilitating a higher sampling efficiency than $\mathrm{MCL}$, it has the same filtering requirements as MCL and is therefore much slower than NCMCL. Here, NCMCL adopts a sample of fixed size from the area, and the extracted sample is not filtered, which makes sampling NCMCL just run it again, resulting in a greatly reduced computational complexity than the other two positioning methods. These results demonstrate that NCMCL provides simpler operation and more accurate positioning results than the other two positioning methods, which makes it better adapted to low-power requirements.

\subsection{Interpretation of results}

Location data associated with WSNs represents critical information. Current developments in localization algorithms employed with WSNs seek to adopt positioning methods that are more robust and stable, and more accurate and efficient while requiring a minimum of resources. This paper proposed the NCMCL method for providing accurate positioning ideally suited to underground mining environments. Simulation results conducted within the narrow roadway setting of a coal mine demonstrated that the proposed algorithm provided greatly improved positioning accuracy and positioning stability than MCB and MCL. Moreover, the computational complexity of the algorithm was greatly reduced, and the number of mobile nodes had minimal impact on the required computation time. The NCMCL positioning method is simpler and more accurate than other commonly employed methods, and is therefore more suitable for low-power applications, as well as for implementation within sensor chips.

\section{Abbreviations}

MCB: Monte Carlo localization boxed; MCL: Monte Carlo localization; NCMCL: Narrow-channel Monte Carlo localization; WSN: Wireless sensor network 


\section{Acknowledgments}

This work was supported by the Gansu Natural Science Foundation under Grant No. 160RJZA003

\section{Declaration}

The experimental data materials of this paper adopt the open data of $\mathrm{UCl}$ database and have reasonable access channels. The publication cost of the paper is supported by the funding of scientific research projects.

\section{Authors' contributions}

QJY and YKY contributed to the conception and algorithm design of the study. EZZ and QJY contributed to the analysis of simulation data, and all authors read and approved the final manuscript.

\section{Author's information}

Qingjun Yang received a M.S. degree from University of Electronic Science and Technology and Lanzhou University, in 2008. He is now a doctoral student in the School of Information Science and Engineering, Lanzhou University. His research interests lie in wireless sensor networks and highperformance computing, pattern recognition, and data mining. He is now working in the Qinghai Province Meteorological bureau.

Dong Sheng Ji He was born in December 1980, in Lanzhou of Gansu Province. He received the BS degree from School of Information Science and Engineering, Lanzhou University, in 2007, and the Ma degree from School of Computer and Communication, Lanzhou University of Technology, in 2011. He is now a doctoral student in the School of Information Science and Engineering, Lanzhou University. His research interests include biomedical image analysis, pattern recognition, and data mining. Yukai Yao He was born in December 1975. He received the BS degree from the Department of Computer Science and Technology, Northwest Normal University, in 1997, and the Ma degree from School of Information Science and Engineering, Lanzhou University, in 2011. He is now a doctoral student in the School of Information Science and Engineering, Lanzhou University. His research interests include high-performance computing, pattern recognition, and data mining. He is a member of CCF and IET. Xiaoyun Chen is professor and a doctoral supervisor of School of Information Science and Engineering, Lanzhou University. She researches database, data mining, high-performance computing, and complex network analysis. She is the director of the Computer Software Institute, Lanzhou University, of the National Center for Linux Technical Training and Promotion of Lanzhou University, and of the center for IBM technology and is a senior member of the CCF, Information Storage Technology Professional Committee, the Ministry of Education Arts Computer Basis Teaching Steering Committee Members. (E-mail:chenxy@lzu.edu.cn).

\section{Competing interests}

The authors declare that they have no competing interests.

\section{Publisher's Note}

Springer Nature remains neutral with regard to jurisdictional claims in published maps and institutional affiliations.

\section{Author details}

${ }^{1}$ School of Information Science\& Engineering, Lanzhou University, Lanzhou 730000, China. ${ }^{2}$ School of Computer and communication, Lanzhou University of Technology, Lanzhou 730000, China. ${ }^{3}$ Qinghai meteorological bureau, Xining 810000, China.

Received: 24 May 2018 Accepted: 1 October 2018

Published online: 23 October 2018

\section{References}

1. D. Niculescu, B. Nath, Ad hoc positioning system (APS). leee 5, 29262931 (2001)

2. D. Niculescu, B. Nath, DV based positioning in ad hoc networks. Telecommun. Syst. 22, 267-280 (2003)

3. O.S. Oubbati, A. Lakas, A survey on position-based routing protocols for Flying Ad hoc Networks (FANETs), vehicular communications, vol Volume 10 (2017), pp. $29-56$
4. S. Boussoufa-Lahlah, A position-based routing protocol for vehicular ad hoc networks in a city environment. Procedia Computer Science 73, 102108 (2015)

5. Y. Shang, W. Ruml, Y. Zhang, M.P.J. Fromherz, Localization from mere connectivity, ACM (2003), pp. 201-212

6. M. Kirk, H. Jane, O. Royan, Deploying a wireless sensor network in iceland Proc of thd 3rd Int Conf on Geosensor Networks (Springer, Berlin, 2009), pp. 131-137

7. L. Hu, D. Evans, Localization for mobile sensor networks, Proceedings of the 10th annual international conference on Mobile computing and networking (ACM, Philadelphia, 2004), pp. 45-57

8. A. Baggio, K. Langendoen, Monte Carlo localization for mobile wireless sensor networks. Ad Hoc Netw. 6, 718-733 (2008)

9. H. Victor, Perez-Gonzalez, Relational position location in ad-hoc networks. Ad Hoc Networks Volume 24(Part A), 20-28 (2015)

10. N. Asis, C. Robert, et al., Wireless sensor network for substation monitoring: design and deployment. PROC of the 6th Int conf on Embedded Networked Sensor Systems (ACM, New York, 2008), pp. 365-370

11. T. Qiu, N. Chen, Heterogeneous ad hoc networks: architectures, advances and challenges, vol 55 (2017), pp. 143-152

12. A. Harter, A. Hopper, P. Steggles, A. Ward, P. Webster, The anatomy of a context-aware application. Wirel. Netw 8, 187-197 (2002)

13. L. Girod, D. Estrin, A reliable routing protocol for vehicular ad hoc networks. Computers \&Electrical Engineering 64, 473-495 (2017)

14. R. Alsaqour, M. Abdelhaq, Dynamic packet beaconing for GPSR mobile ad hoc position-based routing protocol using fuzzy logic. J. Netw. Comput. Appl. 47, 32-46 (2015)

15. D. Niculescu, B. Nath, Ad hoc positioning system (APS) using AOA. leee 3, 1734-1743 (2003)

\section{Submit your manuscript to a SpringerOpen ${ }^{\circ}$ journal and benefit from:}

- Convenient online submission

- Rigorous peer review

- Open access: articles freely available online

High visibility within the field

- Retaining the copyright to your article

Submit your next manuscript at $>$ springeropen.com 